\title{
X-RAY EMISSION FROM PROTOSTELLAR JET HH 154: THE FIRST EVIDENCE OF A DIAMOND SHOCK?
}

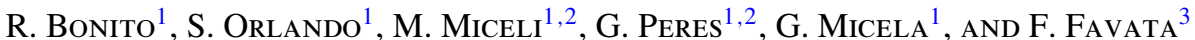 \\ ${ }^{1}$ INAF-Osservatorio Astronomico di Palermo, P.zza del Parlamento 1, 90134 Palermo, Italy; sbonito@ astropa.unipa.it \\ 2 Dipartimento di Fisica-Specola Astronomica, Università di Palermo, P.zza del Parlamento 1, 90134 Palermo, Italy \\ ${ }^{3}$ European Space Agency Community Coordination and Planning Office, 8-10 rue Mario Nikis, F-75738 Paris Cedex 15, France \\ Received 2011 March 31; accepted 2011 May 19; published 2011 August 2
}

\begin{abstract}
$\mathrm{X}$-ray emission from about 10 protostellar jets has been discovered and it appears as a feature common to the most energetic jets. Although X-ray emission seems to originate from shocks internal to jets, the mechanism forming these shocks remains controversial. One of the best-studied X-ray jets is HH 154, which has been observed by Chandra over a time base of about 10 years. We analyze the Chandra observations of HH 154 by investigating the evolution of its X-ray source. We show that the X-ray emission consists of a bright stationary component and a faint elongated component. We interpret the observations by developing a hydrodynamic model describing a protostellar jet originating from a nozzle and compare the X-ray emission synthesized from the model with the $\mathrm{X}$-ray observations. The model takes into account the thermal conduction and radiative losses and shows that the jet/nozzle leads to the formation of a diamond shock at the nozzle exit. The shock is stationary over the period covered by our simulations and generates an X-ray source with luminosity and spectral characteristics in excellent agreement with the observations. We conclude that the X-ray emission from HH 154 is consistent with a diamond shock originating from a nozzle through which the jet is launched into the ambient medium. We suggest that the physical origin of the nozzle could be related to the dense gas in which the HH 154 driving source is embedded and/or to the magnetic field at the jet launching/collimation region.
\end{abstract}

Key words: hydrodynamics - Herbig-Haro objects - ISM: jets and outflows - X-rays: ISM

Online-only material: color figures

\section{INTRODUCTION}

Thanks to the capabilities of current X-ray observatories (Chandra and XMM-Newton), a new class of X-ray sources has been discovered in the past few years, namely those associated with protostellar jets. This class consists of about 10 members, HH 154 being one of the best studied. The X-ray source associated with $\mathrm{HH} 154$ has been previously observed by XMM-Newton (Favata et al. 2002) and by Chandra (Bally et al. 2003; Favata et al. 2006). These observations have shown that the X-ray source is stationary over a period of four years with a possible transient and faint component in the 2005 Chandra observations (Favata et al. 2006).

Hydrodynamic models of the interaction between a continuously ejected supersonic jet and the ambient medium predict $\mathrm{X}$-ray emission caused by shocks at the interaction front (Bonito et al. 2004, 2007). X-ray emission close to the base of the jet is predicted in the case of a pulsed-jet (Bonito et al. 2010a, $2010 \mathrm{~b})$. The pulsed-jet model is very effective in reproducing the features and variability observed in most of the X-ray emitting jets from low-mass young stellar objects (Bonito et al. 2010a). In the pulsed-jet scenario, Bonito et al. (2010a) provided predictions on future observations of $\mathrm{HH} 154$ and claimed that a stationary $\mathrm{X}$-ray source on a period of $\approx 10$ years is unlikely. For a stationary X-ray source, they suggested that a nozzle formed by either a dense medium or a magnetic field may be at work at the launching site, leading to the formation of a stationary diamond shock at the base of the jet.

The new Chandra observation of HH 154 (collected in 2009) provides a time base of eight years to analyze the spectral and morphological variability of the X-ray source. Here we analyze the 2009 observations and compare the results of the data analysis with the previous Chandra (Bally et al.
2003; Favata et al. 2006) and XMM/Newton (Favata et al. 2002) observations. We have developed a model describing a protostellar jet outflowing from a nozzle and compare the $\mathrm{X}$-ray emission synthesized from the model with the Chandra observations. Finally, we interpret the new and the previous observations in light of our model results. In Section 2, we describe the Chandra observations and their analysis; in Section 3, we discuss the hydrodynamic model of jet/nozzle and the comparison between model predictions and observations; and in Section 4, we discuss the results and draw our conclusions.

\section{CHANDRA OBSERVATIONS OF HH 154}

We present the new Chandra/ACIS-S data of HH 154 (PI Schneider; ObsID 11016; $t_{\exp }=65.2 \mathrm{ks}$ ) centered at (04:31:34.998, + 18:07:51.95) (FK5), performed on 2009 December 29, that provides eight years of time base between the first (2001; Favata et al. 2002) and the last Chandra observations of $\mathrm{HH} 154$, with the intermediate observation in 2005 (Favata et al. 2006). To derive a uniform comparison between the three Chandra data sets, we reprocessed all the data with the same method, using the latest CIAO 4.3 package, and filtered the data to restrict the energy to the $0.3-4.0 \mathrm{keV}$ band, as in Favata et al. (2006). Events were extracted for all observations from regions around the source and the background, near the position of the HH 154 jet (4:31:34.10, + 18:08:04.9), following Bally et al. (2003). We defined a box $3.5 \times 5^{\prime \prime}$ in the 2001 data set, a box $3^{\prime \prime} .5 \times 5^{\prime \prime} .5$ in the 2005 data set, and a box $3^{\prime \prime} \times 6^{\prime \prime}$ in the 2009 data set ${ }^{4}$ to extract the source events, and four 10 pixel radius circles for the background, from source-free parts of the image around the source. We extracted the spectra of the three

\footnotetext{
4 The size of the boxes is different for different years to account for the variation of the morphology of the X-ray source.
} 

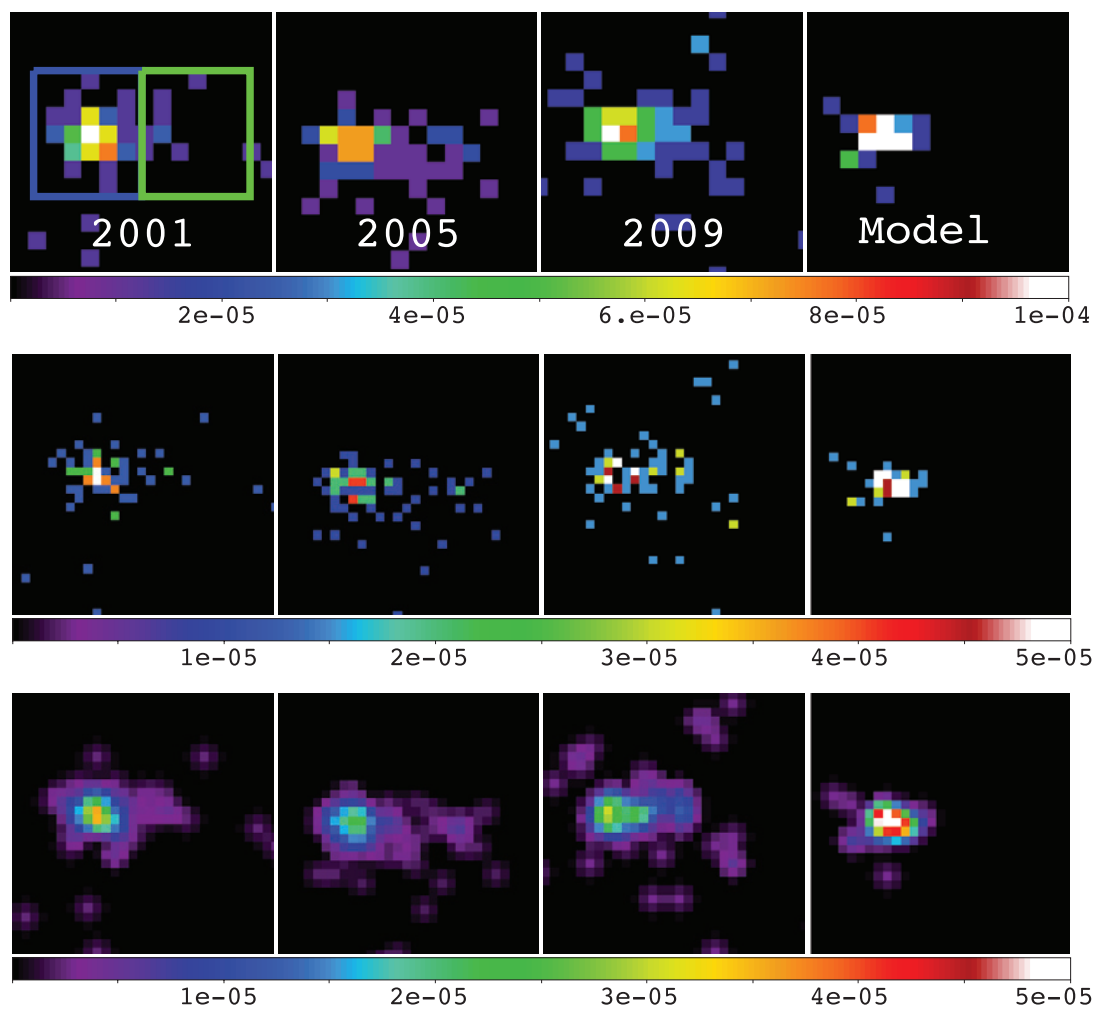

Figure 1. Upper panels: X-ray count rate maps (0.3-4 keV) of the 2001, 2005, and 2009 data sets (first to third panels) at the native ACIS pixel size (0'.5). The last panel shows the synthetic X-ray map of the base of the jet as derived from the model at the same spatial resolution as Chandra/ACIS. Central panels: the same as upper panels, but resampled at $0^{\prime \prime} 25$. EDSER technique has been applied on these data as explained in the text. Lower panels: the same as central panels, but with a smoothing applied on the images (Gaussian kernel of width 0.5). In each panel north is up and east is left. The angular size of each panel is $\approx 7^{\prime \prime} \times 7^{\prime \prime}$. The P.A. of the PSF asymmetry (see explanation in Section 2.1) is $93^{\circ}$ in $2001,102^{\circ}$ in 2005 , and $269^{\circ}$ in 2009 . Note that this asymmetry can produce artificial extension on angular scales up to $1^{\prime \prime}$, as discussed in the text.

(A color version of this figure is available in the online journal.)

data sets and produced the ancillary response files, arf and rmf, by using SPECEXTRACT. We grouped the spectra to have a minimum of 5 counts per bin.

We also applied the sub-pixel repositioning algorithm available in CIAO 4.3 (EDSER) to the Chandra images to refine the event positions ( $\mathrm{Li}$ et al. 2004). We resample the improved images at 0 ' 25 pixel size, obtaining images with one-half of the native ACIS pixel scale. Figure 1 shows the X-ray source associated with HH 154 in 2001 (first panels), 2005 (second panels), and 2009 (third panels), and the X-ray maps synthesized from our hydrodynamic model (discussed in Section 3) with the same spatial resolution as ACIS (last panels). The upper panels show the images with the native ACIS pixel size (0.5); the central panels show the images with sub-pixel repositioning algorithm applied and resampled with a pixel size $0^{\prime} .25$; the lower panels show the improved resampled images smoothed using a Gaussian kernel of width 0.'5.

\subsection{Results}

The three Chandra data sets show that the X-ray emission is mainly located at the base of the HH 154 jet in all epochs, near the driving source. Bally et al. (2003) found that the $\mathrm{X}$-ray source is displaced by $0.5-1^{\prime \prime}$ with respect to the L1551 IRS5 driving source. The X-ray source consists of a bright knot which appears to be stationary in the time period covered by the observations (in the following the "stationary" component) and an elongated structure directed away from the driving source (in the following the "elongated" component) showing variability in the three data sets (see Figure 1). In particular, the latter component appears as a knot in images with EDSER applied (central and lower panels in Figure 1) which is much fainter than the knot of the stationary component. The position of the faint knot appears to be different in the three data sets. In particular, for the 2001 and 2005 data, we confirm the results of Favata et al. (2006), i.e., an elongation of the $\mathrm{X}$-ray source corresponding to a proper motion of 0 !' $7 \mathrm{yr}^{-1}$ approximately westward away from the driving source of the HH 154 jet. As for the 2009 observations, there is a hint of a faint knot closer to the brighter stationary source than in 2005. Note that recently, an asymmetry in the Chandra point spread function (PSF) has been discovered, ${ }^{5}$ located at position angle P.A. $=195-$ roll angle $( \pm 25)^{\circ}$, corresponding to P.A. $=269^{\circ} .4$ in the 2009 observations, i.e., roughly the direction of the extension of the X-ray source detected in HH 154. We checked if this instrumental effect may influence the observed morphology of the X-ray source and found that the asymmetry of the PSF does not affect our images on scales larger than 1 arcsec. The elongated structure visible in the images, therefore, is not an artifact of the instrument. The maximum length of the whole $\mathrm{X}$-ray source is $\approx 5^{\prime \prime}(\approx 700 \mathrm{AU}$ at $D=140 \mathrm{pc})$.

We performed a spectral analysis of the individual data sets. ${ }^{6}$ All spectra are well fitted by an absorbed thermal plasma (APEC in XSPEC). The count rates of the three observations $\left(0.76 \pm 0.10\right.$ counts ks ${ }^{-1}$ in $2001,0.65 \pm 0.08$ counts ks ${ }^{-1}$ in

\footnotetext{
5 See http://cxc.harvard.edu/ciao/caveats/psf_artifact.html for details.

6 We have verified that our procedure reproduces the results obtained by Bally et al. (2003) and Favata et al. (2006).
} 


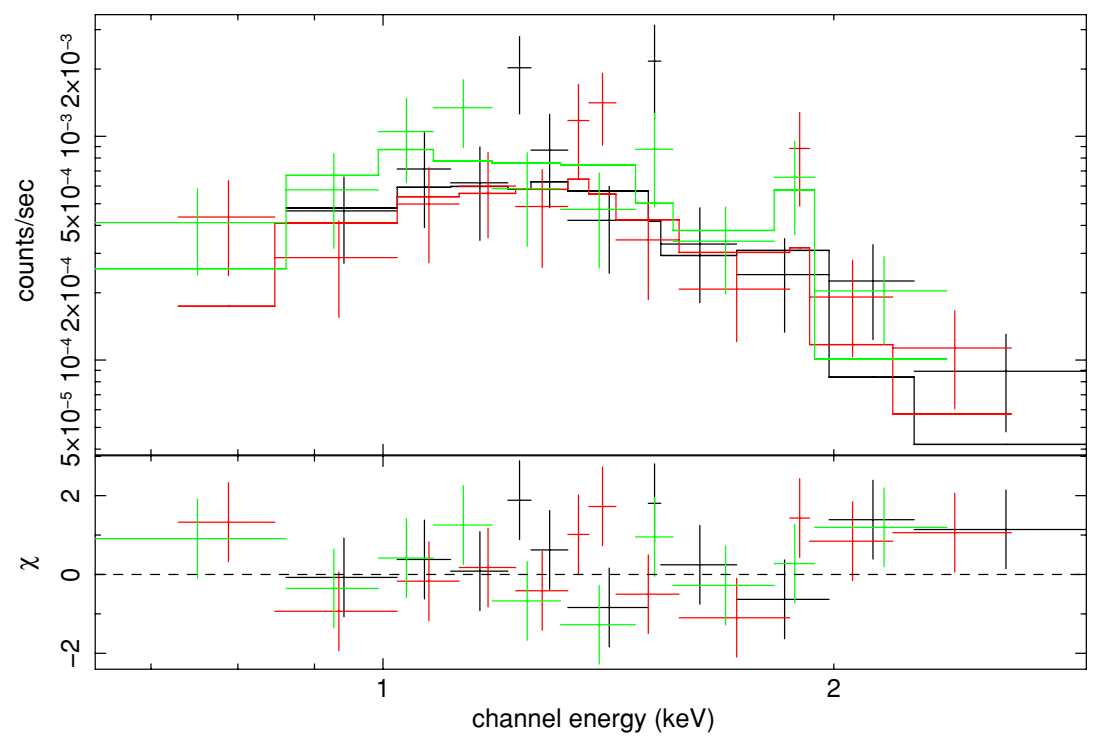

Figure 2. Best-fit X-ray spectral model superimposed on the three Chandra data sets (2001 in black, 2005 in red, and 2009 in green) fitted simultaneously.

(A color version of this figure is available in the online journal.)

Table 1

Best-fit Values Derived from the Three Data sets Simultaneously Fitted

\begin{tabular}{|c|c|c|c|c|}
\hline $\begin{array}{l}T \\
(\mathrm{~K})\end{array}$ & $\begin{array}{c}E M \\
\left(\mathrm{~cm}^{-3}\right)\end{array}$ & $\begin{array}{c}F_{X} \\
\left(\mathrm{erg} \mathrm{cm}^{-2} \mathrm{~s}^{-1}\right)\end{array}$ & $\begin{array}{c}L_{X} \\
\left(\mathrm{erg} \mathrm{s}^{-1}\right)\end{array}$ & $\begin{array}{c}N_{\mathrm{H}} \\
\left(\mathrm{cm}^{-2}\right)\end{array}$ \\
\hline $7_{-2}^{+8} \times 10^{6}$ & $5_{-3}^{+4} \times 10^{51}$ & $5_{-3}^{+4} \times 10^{-14}$ & $1.3_{-0.9}^{+0.8} \times 10^{29}$ & $1.2 \pm 0.2 \times 10^{22}$ \\
\hline
\end{tabular}

2005, and $0.89 \pm 0.12$ counts $\mathrm{ks}^{-1}$ in 2009) are compatible within the Poisson error, indicating that the X-ray luminosity is constant. Moreover, the source shows no spectral variability in terms of temperature $(T)$ and emission measure $(E M)$ in the timescale analyzed here. We have thus fit the three data sets simultaneously, finding the values reported in Table 1.

The $N_{\mathrm{H}}$ is well constrained (more than in the analysis of XMM-Newton data; Favata et al. 2002) by fitting the three data sets simultaneously but it cannot be constrained by the individual data sets. In the latter case, we fixed $N_{\mathrm{H}}$ to the value derived in the simultaneous fitting of the three data sets. The three data sets and the best-fit model are shown in Figure 2.

To investigate possible spatial variations of the spectral properties, we selected two regions, confining the stationary component (blue box in Figure 1) and the elongated component (green box in Figure 1), and computed the median photon energy, MPE, within each region in 2001, 2005, and 2009. MPE has been proved to be a robust indicator of the spectral properties of a source in the case of low statistics (Hong et al. 2004). In each data set the total number of counts considered is $\approx 50$, in the stationary component, and $\sim 10$, in the elongated component. The stationary component shows no temporal variability in the three epochs, its MPE varying in the range $\mathrm{MPE}_{\mathrm{s}}=1.35-1.42 \mathrm{keV}$. The median energy of the elongated component $\mathrm{MPE}_{\mathrm{e}}$ is always lower than $\mathrm{MPE}_{\mathrm{s}}$. By considering the three observations altogether, $\mathrm{MPE}_{\mathrm{s}} \approx 1.4 \mathrm{keV}$, while the elongated component appears softer with $\mathrm{MPE}_{\mathrm{e}} \approx 1.0 \mathrm{keV}$.

This result is supported by the Kolmogorov-Smirnov test we performed to check if the photon energy distributions of the two regions differ significantly and vary with time. We found that the stationary and the elongated component are compatible with being constant over the time base analyzed. We also found that the two components are dissimilar at the $99.99 \%$ confidence level. In light of this result, we have fitted simultaneously the data collected in the three epochs for each of the two components and found that the stationary component is described by a plasma with $T=(7-14) \times 10^{6} \mathrm{~K}$ and the elongated component by a plasma with $T<7 \times 10^{6} \mathrm{~K}$ (assuming the same $N_{H}$ for the two spectra), confirming that the elongated component is softer.

\section{THE MODEL}

X-ray observations of HH 154 suggest the presence of a steady shock at the base of the jet over a time interval of about eight years. Examples of quasi-stationary shocks in jets are the diamond shocks, namely stationary shock patterns appearing in supersonic jets when the jet material outflowing from a nozzle is slightly over- or underexpanded or, in other words, when the pressure of the gas exiting the nozzle is below or above the pressure of the ambient medium. Bonito et al. (2010a) suggested that a diamond shock forming near the launching/collimation site of the jet is the most likely mechanism leading to a stationary X-ray source in HH 154.

To test the above idea, we extended the hydrodynamic model of Bonito et al. (2007; to which the reader is referred for more details), describing the interaction between a continuously ejected supersonic jet with the unperturbed medium, by adding a diverging nozzle at the base of the jet included as an impenetrable body (see Figure 3). The model is described by the hydrodynamic equations solved in two dimensions (adopting cylindrical coordinates and assuming axisymmetry with the jet axis coincident with the axis of symmetry; Bonito et al. 2007) and takes into account the thermal conduction (including heat flux saturation) and radiative losses from optically thin plasma. The calculations were performed using FLASH, a welltested adaptive mesh refinement multiphysics code (Fryxell et al. 2000).

The jet with temperature $T_{\mathrm{j}}=10^{4} \mathrm{~K}$ outflows from the nozzle and propagates through an initial uniform ambient medium with the same temperature. The initial jet radius depends on the nozzle size, chosen accordingly with observations of the order of tens of AU (see Bally et al. 2003). We have explored a wide space of model parameters defined by the particle number density of the jet ranging between $n_{\mathrm{j}}=3 \times 10^{2}$ and $5 \times 10^{3} \mathrm{~cm}^{-3}$, the 


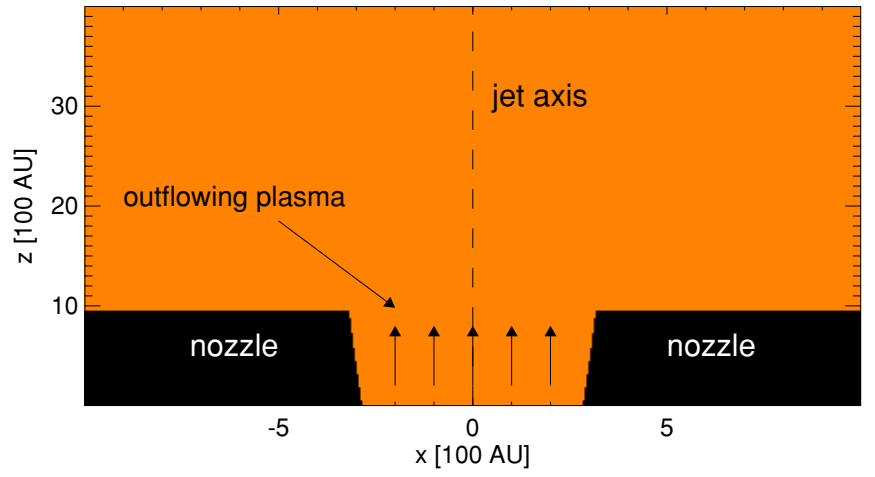

Figure 3. Enlargement of the nozzle through which the jet is ejected into the ambient medium.

(A color version of this figure is available in the online journal.)

density of the ambient medium ranging between $n_{\mathrm{a}}=3 \times 10^{3}$ and $10^{5} \mathrm{~cm}^{-3}$, the throat of the nozzle with radius ranging between $R_{\mathrm{th}}=15$ and $200 \mathrm{AU}$, and the jet velocity ranging between $u_{\mathrm{j}}=1000$ and $1500 \mathrm{~km} \mathrm{~s}^{-1}$. We assume that the direction of propagation of the jet is perpendicular to the line of sight. Our best-fit model is characterized by $n_{\mathrm{j}}=3 \times 10^{2} \mathrm{~cm}^{-3}$, $n_{\mathrm{a}}=3 \times 10^{3} \mathrm{~cm}^{-3}, u_{\mathrm{j}}=1500 \mathrm{~km} \mathrm{~s}^{-1}$, and $R_{\mathrm{th}}=100$ AU. The jet is overexpanded and travels through an initially denser ambient medium (light jet scenario; see Bonito et al. 2007) with ambient-to-jet density contrast $v=n_{a} / n_{j}=10$.

The computational domain is $(1000 \times 4000)$ AU $(\approx 7 \times$ $27 \operatorname{arcsec}$ at $D=140 \mathrm{pc})$. Inflow boundary conditions are imposed at $z=0$ and $r<R_{\text {th }}$, axisymmetric boundary conditions are imposed along the jet axis (consistent with the adopted symmetry), and outflow boundary conditions are assumed elsewhere. The maximum spatial resolution achieved by our simulations is $\approx 2 \mathrm{AU}$, using five refinement levels with the PARAMESH library (MacNeice et al. 2000), which corresponds to covering the initial jet radius of our best-fit model with 50 grid points. The grid resolution is increased inside the nozzle and around the nozzle exit to capture the diamond structure forming there. Note that the pixel size of the hydrodynamic model at its maximum spatial resolution (2 AU) corresponds to a spatial resolution of $0^{\prime \prime} .014$ at the distance of $140 \mathrm{pc}$, i.e., much lower than the nominal spatial resolution of ACIS (0.5). As discussed below, this implies that the synthetic maps derived from the hydrodynamic model needs to be rebinned to match the ACIS pixel size in order to compare the model results with the observations.

\subsection{Synthesis of X-Ray Emission}

Following Bonito et al. (2007), we synthesized the X-ray emission associated with the jet from the model results, recovering the three-dimensional spatial distributions of mass density and temperature. We then derived the emission measure, EM, and temperature, $T$, for each fluid element and the distribution $\operatorname{EM}(T)$ integrated along the line of sight, for each element, in the temperature range $10^{3}-10^{8} \mathrm{~K}$ (using 75 bins equispaced in $\log T)$. From $\operatorname{EM}(T)$, we synthesize the maps of the X-ray emission and the focal plane spectra using the APEC spectral code, and considering photon count statistics comparable with that of the observations. The source is assumed to be at a distance $D=140 \mathrm{pc}$. We filtered the emission through the Chandra/ ACIS instrumental response and the interstellar column density at the best-fit value $N_{H}=1.2 \times 10^{22} \mathrm{~cm}^{-2}$ (see Section 2.1). In particular, to compare the synthesized images with the different observations, we have used the rmf and arf files generated for the three data sets $(2001,2005$, and 2009) to account for the correction to the charge transfer inefficiency and the degradation in low-energy response due to contaminant buildup on the optical blocking filter.

To directly compare the synthesized images with the observations, we rebinned the model images (whose spatial resolution is 2 AU, corresponding to $0^{\prime \prime} 014$ at $140 \mathrm{pc}$ ) so as to have the same bin size as the Chandra images as shown in Figure $1(0.25$, or $0.5)$.

We also convolved the synthesized X-ray image with the specific PSF, created at the proper energy for each data set, by using the Chandra standard analysis tools. We finally included Poisson fluctuations to mimic the photon count statistics.

\subsection{Results}

Figure 4 shows the spatial distribution of density in a slice in the $(x, z)$-plane (left panel) for the best-fit model at time $t \approx 120 \mathrm{yr}$ and a close up view of the nozzle site (bottom right panel). After the jet exits the nozzle, it propagates through the ambient medium forming a shock with temperature $T \approx 10^{7} \mathrm{~K}$ at its head and a cold cocoon enveloping it. The cocoon is characterized by low temperatures $\left(T<10^{5} \mathrm{~K}\right)$, is dominated by the radiative cooling, and is strongly perturbed by the hydrodynamic instability developing there as the jet progresses through the medium, the thermal conduction being inefficient in damping the instability (see Bonito et al. 2010a, 2010b).

The nozzle determines a diamond-shaped shock past the nozzle exit with peak temperature $T \approx 8 \times 10^{6} \mathrm{~K}$ (see right bottom panel in Figure 4). This diamond structure has its origin inside the nozzle and appears as a shock emerging from the nozzle and reflecting just past the nozzle exit. After its formation $(t \approx 50 \mathrm{yr}$ ), the diamond shock is almost stationary until the end of the simulation for $\approx 100 \mathrm{yr}$. Thermal conduction is rather efficient in the post-shock region given the high temperatures there $\left(T>10^{6} \mathrm{~K}\right)$ and is crucial in stabilizing the diamond structure, damping the hydrodynamic instability developing past the nozzle exit. Auxiliary simulations performed without the thermal conduction have shown that the diamond shock would be unstable if the thermal conduction is neglected, the hydrodynamic instability heavily perturbing the flow structure at the nozzle exit.

Analyzing the X-ray emission synthesized from the hydrodynamic model, as described in Section 3.1, we investigated both the morphology and spectral properties of the synthetic X-ray sources. The X-ray emission from the modeled jet consists of two main features: a quasi-stationary source associated with the diamond shock at the jet base and a moving source associated with the shock at the head of the jet. The latter is a transient feature we are not interested in ${ }^{7}$ and does not influence the evolution of the diamond shock at the base of the jet; therefore, we will not discuss its properties in the following.

The X-ray luminosity of the diamond shock is $L_{X} \approx 5 \times$ $10^{29} \mathrm{erg}$ and is stationary over $\approx 100 \mathrm{yr}$. This value is similar to that observed for $\mathrm{HH} 154$, which is almost stationary in about eight years.

By comparing the total flux derived from the model with the specific $\mathrm{rmf}$ and arf response of each data set, we have verified

\footnotetext{
7 The X-ray emission at the head of the jet forms because we simulate the early evolution of the jet when its head is close to the stellar driving source. We expect that the head of the jet progressively cools down because of radiative cooling as it goes away from the driving source, eventually emitting mainly in the optical band.
} 


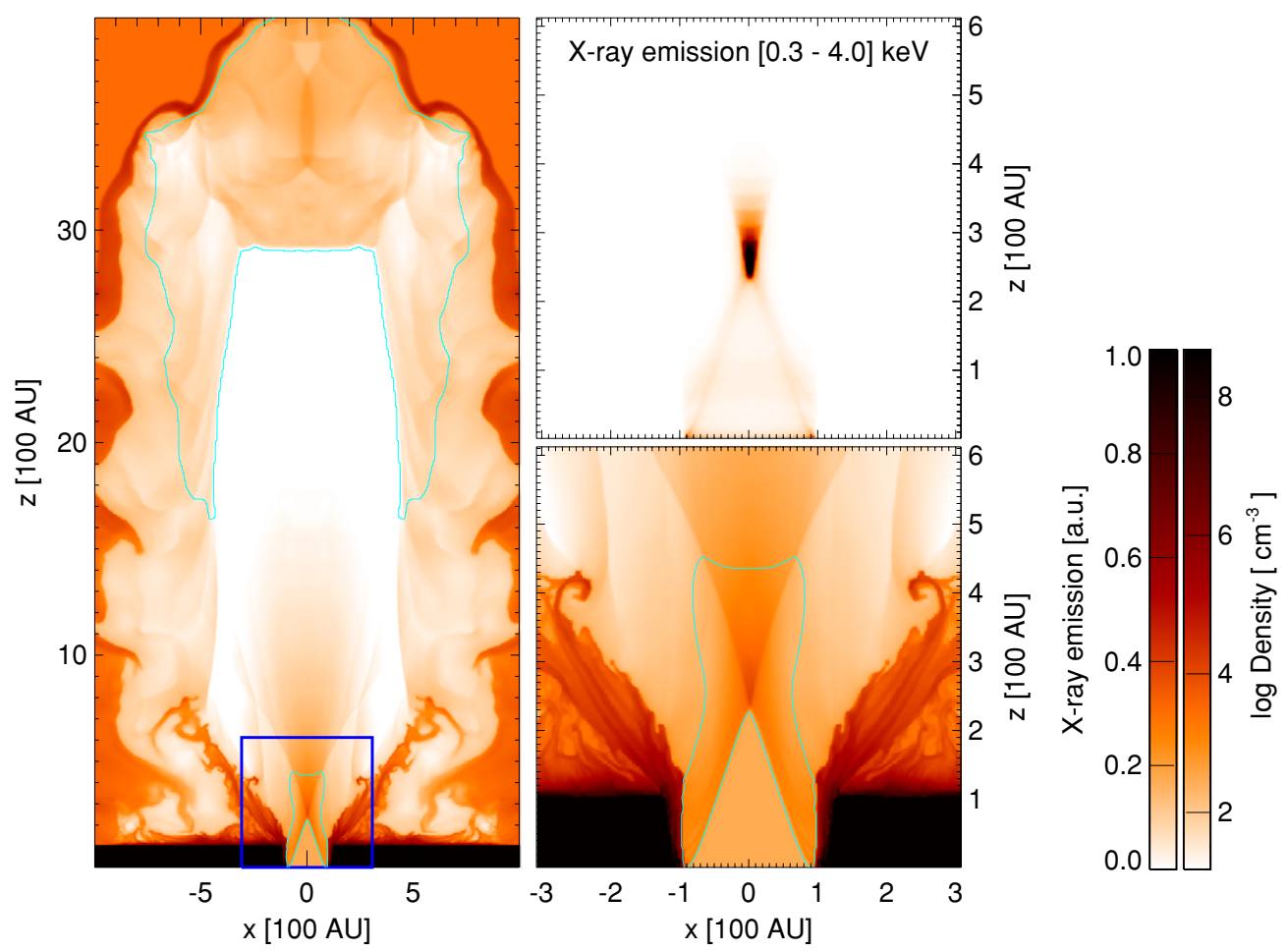

Figure 4. Density map (left panel) with the $2 \times 10^{6} \mathrm{~K}$ contour superimposed, an enlargement of the base of the computational domain (lower panel on the right), and the X-ray map synthesized from the model (upper panel on the right) at the maximum spatial resolution achieved from the simulation. $100 \mathrm{AU}$ correspond to $\approx 0$ '. 7 at $140 \mathrm{pc}$.

(A color version of this figure is available in the online journal.)

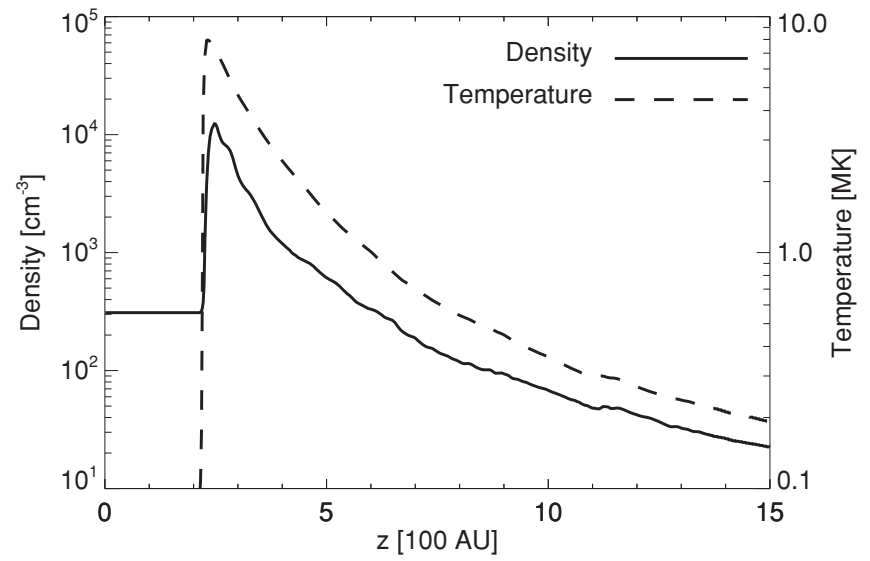

Figure 5. Profiles of particle number density (solid line) and temperature (dashed line) along the jet axis in the region where the diamond shock forms.

that the degraded quantum efficiency of the instrument in the time baseline analyzed affects the synthesized count rate for less than $7 \%$. This confirms that the source flux can be assumed constant over eight years, within the Poisson errors.

The upper right panel in Figure 4 shows the synthetic X-ray emission arising from the shock integrated along the line of sight. Most of the emission originates just behind the shock in a bright and compact knot with temperature $T \approx 8 \times 10^{6} \mathrm{~K}$. The knot is surrounded by a diffuse region elongated along the jet axis, characterized by lower temperatures $\left(T \approx 1-2 \times 10^{6} \mathrm{~K}\right)$. Figure 5 shows the density and temperature profiles along the jet axis in the region where the diamond shock forms.

We found that the spectrum synthesized from the hydrodynamic model, as explained in Section 3.1, can be fitted with one
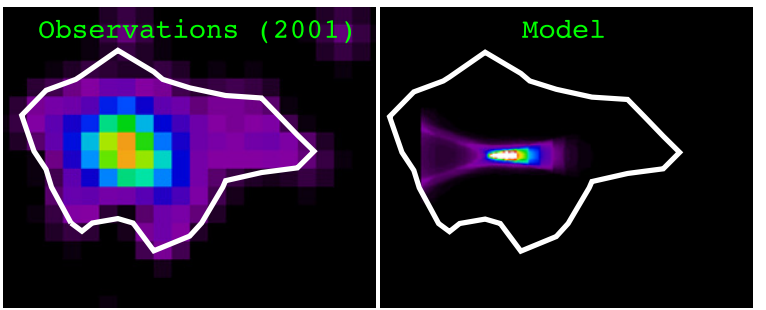

Figure 6. Smoothed 2001 image of the HH 154 X-ray source (left panel) with a pixel size of 0.25 compared with the high-resolution $\left(00^{\prime \prime} 014\right)$ image derived from the model (right panel). The contour of the observed source is superimposed on the images. Since we verified that the direction of the PSF asymmetry in 2001 is along the counter jet and since this asymmetry can produce artificial structures only out to $1^{\prime \prime}$ (four pixels at the 0 '.25 pixel size of the image on the left panel), the observed elongation is not an artifact due to the PSF asymmetry. The angular size of each panel is $\approx 5^{\prime \prime} \times 4^{\prime \prime}$. In both panels, north is up and east is left.

(A color version of this figure is available in the online journal.)

isothermal component compatible with that derived from the three data sets of Chandra.

We rescaled the synthetic X-ray image shown in Figure 4 to the Chandra/ACIS pixel size (last panels in Figure 1). The emission within the nozzle is assumed to be totally absorbed. We found that the spatial scales of the X-ray emitting diamond shock at the same spatial resolution of Chandra are consistent with the size of the HH 154 X-ray emitting source: a synthetic $\mathrm{X}$-ray source of a few arcsec at the base of the jet consisting of a bright point-like component surrounded by a faint and elongated component along the jet axis.

In Figure 6, we compare the smoothed 2001 image with a bin size of $0^{\prime \prime} .25$ (left panel) with the X-ray source derived from the 
model at its maximum spatial resolution, 0.'014 (right panel). The 2001 image contour is superimposed on the modeled source.

\section{DISCUSSION AND CONCLUSIONS}

The analysis of the observations of HH 154 in three different epochs with Chandra reveals a faint and elongated X-ray source displaced by $0.5-1$ arcsec (Bally et al. 2003) from the L1551 IRS 5 protostar (the driving source of the jet) along the jet axis. The source appears to be quasi-stationary over a time base of $\approx 8 \mathrm{yr}$ without appreciable proper motion and variability of $\mathrm{X}$-ray luminosity and of temperature. The morphological analysis shows that the X-ray source consists of a bright stationary component with temperature $T>7 \times 10^{6} \mathrm{~K}$ surrounded by an elongated cooler component extended in the direction away from the driving source, with temperatures $T<7 \times 10^{6} \mathrm{~K}$. Very recently, Schneider et al. (2011) analyzed the same data sets, finding similar observational results in terms of X-ray luminosity, spectral parameters, and morphology, independently showing the robustness of the derived parameters that form the basis of our comparison with a simulation of the jet based on detailed hydrodynamic models.

As shown in Bonito et al. (2008), the X-ray source is not perfectly aligned with the optical jet observed in HH 154 (see Figure 13 in Bonito et al. 2008). In fact, the Hubble Space Telescope images of Fridlund et al. (2005) show that the optical jet from HH 154 is along P.A. $\approx 254^{\circ}$ (see also Pyo et al. 2002), while from the X-ray data we derive a P.A. $\approx 270^{\circ}$. Bonito et al. (2010a) suggested that an ejection direction varying in time could explain the misalignment between the X-ray source and the optical jet. Since the jet driving source, L1551 IRS5, is known to be a binary system (Bieging \& Cohen 1985), a jet precession could be induced due to the presence of the companion star.

The absorption column density derived from the analysis of the three data sets is too low if compared with the 150 mag of absorption of L1551 IRS 5, confirming the results of Bally et al. (2003) and Favata et al. (2006). This fact together with the evident displacement of $0.5-1^{\prime \prime}$ of the source from L1551 IRS 5 and the lack of temporal variation in the X-ray flux and spectral properties suggest that the X-ray emission detected in the three epochs unambiguously arises from the jet and cannot be of stellar origin.

The observations suggest therefore that the X-ray emission of $\mathrm{HH} 154$ originates in a standing shock located at the base of the jet. Bonito et al. (2010a), by analyzing the X-ray emission arising from a pulsed jet model, have discussed the possibility to produce a standing shock at the base of the jet as a result of multiple self-interactions of plasma blobs ejected in different epochs by the driving source and concluded that this mechanism is unlikely. In addition, these authors suggested that the most likely mechanism leading to a standing shock over a period $>5 \mathrm{yr}$ might be a diamond shock forming near the launching/ collimation site of the jet.

This idea is expanded here by developing a model of jet outflowing through a nozzle. We found that, in such a configuration, a standing diamond shock forms just past the nozzle exit at the base of the jet. The shock is stabilized under the action of the thermal conduction which damps the hydrodynamic instability developing within the cocoon and heavily perturbing the flow structure. We found that the X-ray emission arising from the diamond shock has morphology and spectral characteristics in excellent agreement with those derived from the three data sets of HH 154. The model also predicts that the X-ray emitting plasma of the diamond shock cools down at larger distances from the driving source as inferred from the observations. In fact, from the Chandra data, we found that the MPE increases toward the base of the jet. While we cannot rule out that this result is associated with variations in $N_{H}$ (Fridlund et al. 2005 found an increasing absorption toward the driving source along the jet axis), higher values of MPE can be indicative of higher temperatures. Such variations of plasma temperature would be naturally explained by our model. We conclude therefore that HH 154 offers the first evidence of a standing diamond shock at the base of the jet probably near the jet launching/collimation region.

We can infer a characteristic size, $l_{\mathrm{sh}}$, of the X-ray emitting source from the spectral analysis, using the value of $\mathrm{EM}_{\text {best-fit }}$, and from the hydrodynamic model results, using the maximum particle density value derived from the diamond shock modeled. In particular, we find $l_{\mathrm{sh}}>V^{1 / 3}=4 \times 10^{14} \mathrm{~cm}$, where $V$ is the volume derived from EM $=0.8 n^{2} V$ (following Favata et al. 2002) and $n_{\mathrm{MAX}} \approx 10^{4} \mathrm{~cm}^{-3}$ (see the peak of density in Figure 5) at $140 \mathrm{pc}$. This value is in good agreement with both the observed radius of $\mathrm{HH} 154, r_{j} \approx 30 \mathrm{AU}$ (see discussion on this parameter in both models and observations in Bonito et al. 2007, 2008), and with the size of the diamond shock modeled. Therefore, although the spatial resolution of the ACIS data, improved by sub-pixel techniques, is more than an order of magnitude lower than that achieved by our numerical model, its diagnostic power allows us to infer detailed information on physical scales comparable to numerical simulations.

The data, after this analysis, clearly show the presence of an elongated structure on the right side of the main source. Concerning an eventual evolution of this elongated structure the most conservative interpretation could be that it is steady and we can hardly constrain its features, due to the limited photon statistics. However, given the insight provided by the model and the evidence that stellar jet flows are inherently variable, another interpretation is that we are observing the diamond shock variability and possibly knot formation and motion due to the changes of the jet flow (see Bonito et al. 2010a for example of effects of variable jet flows). The sequence of the smoothed images of $\mathrm{HH} 154$ with a spatial scale of 0.25 shown in Figure 1 suggests the presence of subsequent knots with a detectable proper motion. In particular, by comparing the 2001 and 2005 data sets, we confirm the results of Favata et al. (2006) who found a detectable westward proper motion of the elongated component of the X-ray source, corresponding to $\approx 500 \mathrm{~km} \mathrm{~s}^{-1}$; we find a hint of elongation again westward in the 2009 observations away from the jet driving source, but closer to the stationary source than in the 2005 observations. This evidence may suggest the presence of a newly formed knot propagating away from the diamond shock. However note that, in the 2009 observation, the PSF asymmetry is directed almost along the X-ray extension axis, and therefore it could influence the $\mathrm{X}$-ray source elongation up to an angular scale $\approx 1^{\prime \prime}$. Therefore, both the evidence of a standing shock at the base of the $\mathrm{HH} 154$ jet over a 8 yr timebase and a moving knot in 2005 together with a hint of a new elongation in the 2009 smoothed image, indicate the scenario of a nozzle, creating the standing shock, in the presence of a pulsed jet, as described in Bonito et al. (2010a), which may account for the moving/elongated component. No matter how one interprets the observations, there is a clear need for future observations of HH 154. 
The physical origin of the nozzle could be related to the dense gas in which the L1551 IRS 5 protostar is embedded and/or the intense stellar magnetic field at the jet launching/collimation region. In the hypothesis that a magnetic nozzle causes the diamond shock observed in HH 154, the Chandra observations and the comparison with our model offer the possibility to constrain the magnetic field strength near the jet launching/collimation region. In fact, the model provides the total plasma pressure $\left(p_{\mathrm{sh}}+\rho_{\mathrm{sh}} u_{\mathrm{sh}}^{2}\right)$ in the X-ray emitting diamond shock that reproduces the observations, where $p_{\mathrm{sh}}, \rho_{\mathrm{sh}}$, and $u_{\mathrm{sh}}$ are the pressure, mass density, and velocity in the post-shock region close to the nozzle exit, respectively. Then, assuming the plasma $\beta=\left(p_{\mathrm{sh}}+\right.$ $\left.\rho_{\mathrm{sh}} u_{\mathrm{sh}}^{2}\right) /\left(B^{2} / 8 \pi\right) \approx 1$, where $B$ is the magnetic field strength, we derive $B \approx 5 \mathrm{mG}$ in the magnetic nozzle at the base of the jet. Interestingly, this value is consistent with that inferred by Bally et al. (2003), namely $B=1-4 \mathrm{mG}$, in the context of shocks associated with jet collimation, and by Schneider et al. (2011), who find $B \approx 6 \mathrm{mG}$, which is a reasonable value at the jet basis near the driving source, according to Hartigan et al. (2007). We suggest therefore that the comparison between our model and the X-ray observations of HH 154 may allow us to probe the launching/collimation region near the driving source, which is very difficult to be directly observed in systems so obscured.

We thank an anonymous referee for constructive and helpful comments. R.B. thanks Dr. M. Caramazza, Dr. E. Flaccomio, and Dr. G. Sacco for interesting discussions about the CIAO tools. This research has made use of data obtained from the Chandra Data Archive, and software provided by the Chandra X-ray Center (CXC) in the application package CIAO. The software used in this work was in part developed by the DOE-supported ASC/Alliances Center for Astrophysical
Thermonuclear Flashes at the University of Chicago. The calculations were performed at the SCAN (Sistema di Calcolo per l'Astrofisica Numerica) HPC facility of the INAF-Osservatorio Astronomico di Palermo. This work was supported in part by Agenzia Spaziale Italiana under contract No. ASI-INAF $(\mathrm{I} / 009 / 10 / 0)$.

\section{REFERENCES}

Bally, J., Feigelson, E., \& Reipurth, B. 2003, ApJ, 584, 843

Bieging, J. H., \& Cohen, M. 1985, ApJ, 289, L5

Bonito, R., Fridlund, C. V. M., Favata, F., Micela, G., Peres, G., Djupvik, A. A., \& Liseau, R. 2008, A\&A, 484, 389

Bonito, R., Orlando, S., Miceli, M., Eislöffel, J., Peres, G., \& Favata, F. 2010a, A\&A, 517, A68

Bonito, R., Orlando, S., Peres, G., Eislöffel, J., Miceli, M., \& Favata, F. 2010b, A\&A, 511, A42

Bonito, R., Orlando, S., Peres, G., Favata, F., \& Rosner, R. 2004, A\&A, 424, L1

Bonito, R., Orlando, S., Peres, G., Favata, F., \& Rosner, R. 2007, A\&A, 462, 645

Favata, F., Bonito, R., Micela, G., Fridlund, M., Orlando, S., Sciortino, S., \& Peres, G. 2006, A\&A, 450, L17

Favata, F., Fridlund, C. V. M., Micela, G., Sciortino, S., \& Kaas, A. A. 2002, A\&A, 386, 204

Fridlund, C. V. M., Liseau, R., Djupvik, A. A., Huldtgren, M., White, G. J., Favata, F., \& Giardino, G. 2005, A\&A, 436, 983

Fryxell, B., et al. 2000, ApJS, 131, 273

Hartigan, P., Frank, A., Varniére, P., \& Blackman, E. G. 2007, ApJ, 661, 910

Hong, J., Schlegel, E. M., \& Grindlay, J. E. 2004, ApJ, 614, 508

Li, J., Kastner, J. H., Prigozhin, G. Y., Schulz, N. S., Feigelson, E. D., \& Getman, K. V. 2004, ApJ, 610, 1204

MacNeice, P., Olson, K. M., Mobarry, C., de Fainchtein, R., \& Packer, C. 2000, Comput. Phys. Commun., 126, 330

Pyo, T., Hayashi, M., Kobayashi, N., Terada, H., Goto, M., Yamashita, T., Tokunaga, A. T., \& Itoh, Y. 2002, ApJ, 570, 724

Schneider, P. C., Guenther, H. M., \& Schmitt, J. H. M. M. 2011, A\&A, 530, 123 